\title{
Role of neutrinos for the nucleosynthesis of heavy elements beyond iron in explosions of massive stars
}

\section{Tobias Fischer*}

Institute for Theoretical Physics, University of Wroclaw, Plac Maksa Borna 9 ,

50-204 Wroclaw, Poland

E-mail: fischereift.uni.wroc.pl

\section{Gabriel Martínez-Pinedo ${ }^{1,2}$, Andreas Lohs ${ }^{1}$ and Lutz Huther ${ }^{1}$}

1 Institut für Kernphysik (Theoriezentrum), Technische Universität Darmstadt, Schlossgartenstraße 2, D-64289 Darmstadt, Germany

2 GSI Helmholtzzentrum für Schwerionenforschung, Planckstraße 1, D-64291 Darmstadt, Germany

\begin{abstract}
We discuss preliminary results form simulations of the protoneutron star (PNS) deleptonization phase, from core-collapse supernova explosion. Particular focus is on the nucleosynthesis relevant conditions of the neutrino-driven wind ejected from the PNS surface. These are determined from the properties of the neutrinos which diffuse out of the high-density interior. Here we discuss their spectra and flux formation at the level of the opacity and illustrate the resulting evolution of the luminosities and average energies. Initially large spectral differences between electron neutrinos and antineutrinos allow for the production of light neutron capture elements in the region between $\mathrm{Zr}$ and Mo in slightly neutron rich environment. Towards later times, spectral differences reduce continuously and result in neutron deficient conditions. This may in principle allow for the $v p$ process which turns out to be inefficient due to the low luminosities. These preliminary results are consistent with observations of metal-poor stars which are deficient in heavy neutron-capture elements.
\end{abstract}

XIII Nuclei in the Cosmos,

7-11 July, 2014

Debrecen, Hungary

\footnotetext{
*Speaker.
} 


\section{Introduction}

Explosions of massive stars are triggered from the initial implosion of the stellar core, followed by the delayed ejection of the stellar mantle. The gravitational energy gain, several times $10^{53} \mathrm{erg}$, is trapped inside the central protoneutron star (PNS) mainly as neutrinos. Several scenarios of energy liberation from the PNS interior into the low-density layer at its surface have been proposed: magneto-rotational [1], acoustic [2], high-density phase transition to quark matter [3] and the standard scenario due to neutrino heating and cooling [4]. For a recent review about corecollapse supernovae, see ref. [5]. Once the explosion proceeds, mass accretion at the PNS surface ceases and as a consequence the PNS settles into a quasi-stationary state. The entire situation is illustrated in Fig. 1 at the example of a $11.2 \mathrm{M}_{\odot}$ progenitor star, evolved consistently through core collapse, bounce, post bounce mass accretion and explosion in spherical symmetry with the supernova code AGILE-BOLTZTRAN [6]. Since core-collapse supernova explosions cannot be obtained in spherically symmetric models for such iron-core progenitors, here we enhance the neutrino heating/cooling rates in order to trigger the explosion onset. Once the explosion develops we switch back to the standard rates.

The later evolution is determined by the emission of neutrinos of all flavors which diffuse out of the high-density PNS interior towards lower densities and temperatures where they finally decouple from matter at the PNS surface. During this evolution, known as Kelvin-Helmholtz cooling phase, the PNS deleptonizes on a timescale on the order of 10-30 seconds [7, 8]. This epoch of the supernova explosion marks also the time interval during which most of neutrinos will be detected form the next Galactic explosion within a distance of the current detectors sensitivities. It is therefore of paramount interest to predict reliable neutrino luminosities and spectra as well as their evolution from the current models. Note that neutrinos are emitted from the low-density layer of accumulated material at the PNS surface during the generally rather short (several $100 \mathrm{~ms}$ ) accretion phase. Neutrinos decouple at higher densities during the PNS deleptonization phase. This important aspect is related to the weak processes which determine the neutrino spectra and fluxes. Prior to the explosion onset, the neutrino spectra and fluxes are determined from mass accretion where the charged current neutrino absorption processes dominate. The situation changes once the explosion develops, after which mass accretion vanishes at the neutrinospheres and the neutrino spectra and fluxes are determined by diffusion.

Furthermore, the neutrinos from the PNS drive a low-mass outflow during the deleptonization phase, ejected from the PNS surface via continuous neutrino heating known as neutrino-driven wind. It is illustrated in Fig. 1 via the vertical solid green lines showing the evolution of selected mass elements. It has long been studied as posisble site for the synthesis of heavy elements beyond iron [17]. The neutrino-driven wind depends only on the neutrino properties, in terms of their luminosities and spectra as well as on the characteristics of the PNS such as mass and radius [18]. The general nucleosynthesis of the neutrino-driven wind from PNSs relates to an $\alpha$-rich freeze out in either neutron-rich or proton-rich environment. The latter important aspect is determined from weak processes, in particular from the competition of $v_{e}$ and $\bar{v}_{e}$ absorption on neutrons and protons respectively. It is subject of this article and will be further discusses in the following section. In sec. 3 we will present preliminary results from a simulation of the PNS deleptonization phase. 


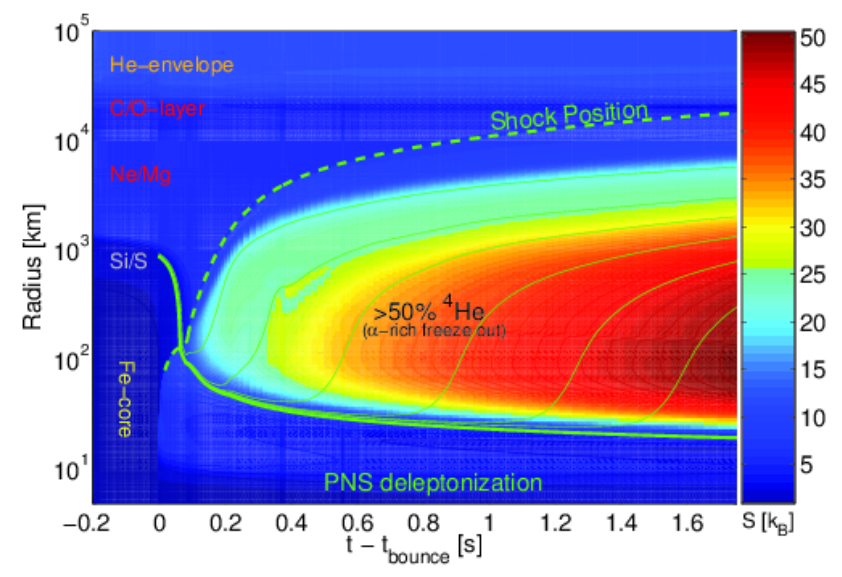

\begin{tabular}{lc}
\hline \hline Weak process & Ref. \\
\hline (1) $v_{e} n \rightleftarrows e^{-} p$ & {$[9]$} \\
(2) $\bar{v}_{e} p \rightleftarrows e^{+} n$ & {$[9]$} \\
(3) $v_{e}(A, Z-1) \rightleftarrows e^{-}(A, Z)$ & {$[10]$} \\
(4) $v N \rightleftarrows v^{\prime} N$ & {$[11,12]$} \\
(5) $v(A, Z) \rightleftarrows v^{\prime}(A, Z)$ & {$[11,12]$} \\
(6) $v e^{ \pm} \rightleftarrows v^{\prime} e^{ \pm}$ & {$[11,13]$} \\
(7) $v \bar{v} \rightleftarrows e^{-} e^{+}$ & {$[11]$} \\
(8) $v \bar{v} N N \rightleftarrows N N$ & {$[14]$} \\
(9) $v_{e} \bar{v}_{e} \rightleftarrows v_{\mu / \tau}+\bar{v}_{\mu / \tau}$ & {$[15,16]$} \\
\hline
\end{tabular}

Figure 1: (left panel) Space-time diagram of a core-collapse supernova explosion. Negative times correspond to the core-collapse phase, gauged at core bounce, $t_{\text {bounce }}$. Color-coding is due to the entropy per baryon, $S$. The shock radius is marked by the green dashed line. The explosion is triggered here at about $\simeq 150 \mathrm{~ms}$ post bounce (infalling Si/S-interface hit the shock) by enhancing the (anti)neutrino absorption rates. We switch back to the standard rates once the explosion proceeds and the central PNS deleptonizes. The high-entropy region above the PNS surface is the neutrino-driven wind. Note that more massive progenitors experiences generally a longer mass accretion phase before explosion onset due to their more extended Si/S-layer above the Fe-core. Table 1: (right panel) Neutrino reactions considered, including references. Note: $v=\left\{v_{e}, \bar{v}_{e}, v_{\mu / \tau}, \bar{v}_{\mu / \tau}\right\}$ unless stated otherwise and $N=\{n, p\} .(A, Z)$ are atomic mass and charge.

\section{Weak processes and nuclear medium effects}

The list of standard weak processes that are considered in core-collapse supernova studies, as well as for the PNS deleptonization phase, are given in Table 1. Weak rates with heavy nuclei, i.e. electron captures, (in)elastic scattering [19], nuclear deexcitations and associated neutrino-pair emission [20], are only relevant when nuclei are abundant. This is only the case during the corecollapse phase and the early post-bounce evolution prior to the explosion onset. These processes play no role during the PNS deleptonization phase. Reactions involving unbound nucleons are of special relevance during the PNS deleptonization phase, in addition to inelastic neutrino scattering on electrons/positrons. The latter process is a major source for the thermalization of the neutrino spectra. For neutrino scattering on nucleons we include inelastic contributions and weak magnetism corrections [21].

The opacity for the charged-current neutrino absorption reactions (1) in Table. 1) is given in ref. [11,9] for which the elastic, i.e. zero-momentum transfer, approximation has been commonly applied:

$$
1 / \lambda\left(E_{v_{e}}\right) \simeq \frac{G_{F}^{2}}{\pi} \frac{V_{u d}^{2}}{(\hbar c)^{7}}\left(g_{V}^{2}+3 g_{A}^{2}\right) p_{e} E_{e}\left(1-f_{e}\left(E_{e}\right)\right) \frac{n_{n}-n_{p}}{1-\exp \left\{\beta\left(\mu_{p}^{0}-\mu_{n}^{0}\right)\right\}},
$$

with $G_{F}$ is the Fermi constant, $g_{V}$ and $g_{A}$ vector and axial-vector coupling constants, and $V_{u d}$ the up-down entry of the Cabibbo-Kobayashi-Maskawa matrix. $f_{e}\left(E_{e}\right)$ is the equilibrium electron Fermi-function. The second term in Eq. (2.1) relates to the nuclear response function of the system to convert a neutron into a proton. Here, for the assumed zero-momentum transfer approximation, 


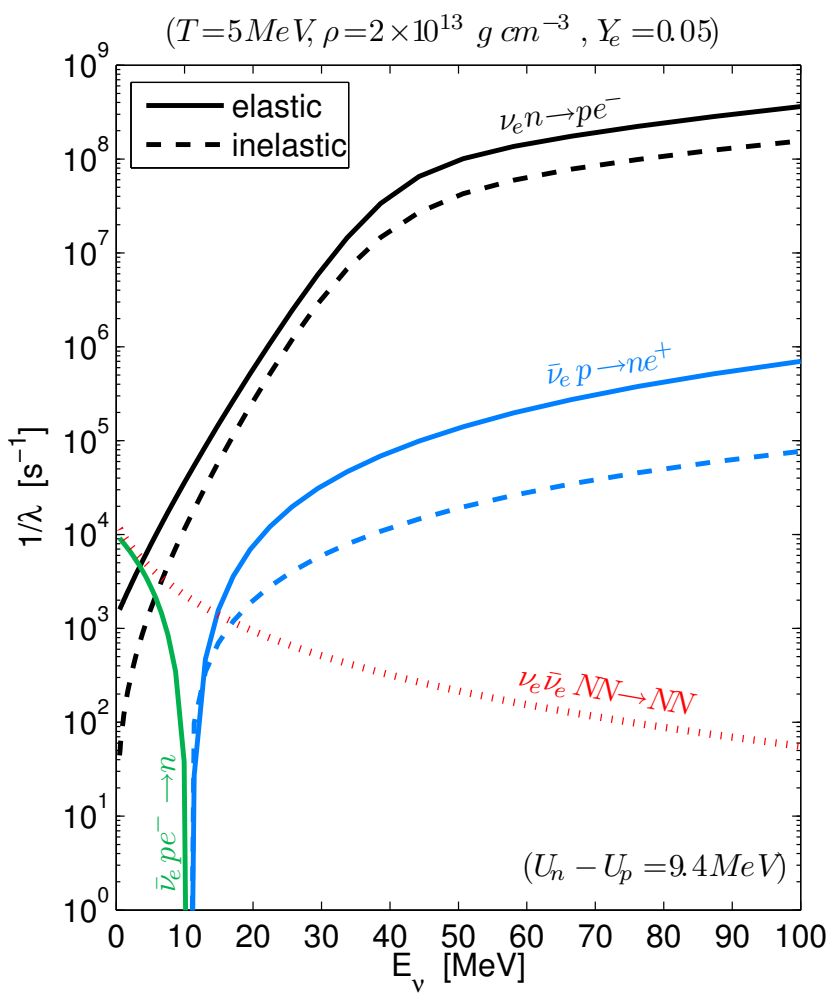

Figure 2: Neutrino opacity for a selection of the relevant weak processes, for the nuclear EOS HS.DD2 [22, 23] at typical conditions for the neutrino decoupling during the PNS deleptonization phase. The nuclear potential difference, $U_{n}-U_{p}=9.4 \mathrm{MeV}$, relates to the modified $Q$-value for the $\bar{v}_{e^{-}}$ absorption opacity (blue solid line), i.e. $Q=Q_{0}+\left(U_{n}-U_{p}\right)$ with $Q_{0}=m_{n}-$ $m_{p}=1.2935 \mathrm{MeV}$. In addition to the elastic approximation, we also show the inelastic charged-current absorption opacity (dashed lines), nucleon-nucleon Bremsstrahlung (red dotted line) and the inverse neutron decay in medium (green dash-dotted line). The opacity for Bremsstrahlung takes into account the $\bar{v}_{e}$ accupation assuming $\bar{v}_{e}$ equilibrium distribution function with chemical potential $\mu_{\bar{v}}=-\mu_{e}+\left(\mu_{n}-\mu_{p}\right)$.

it reduces to the second term in Eq. (2.1) with neutron and proton number densities, $n_{n}$ and $n_{p}$, respectively. A similar expression is obtained for reaction (2) in Table 1, by replacing electron distribution and energy with those of positrons as well as exchanging the labels $n \leftrightarrow p$ in Eq. (2.1). For the reverse reactions, i.e. the neutrino emissivity, detailed balance is applied as follows,

$$
j_{v_{e}}\left(E_{v_{e}}\right)=\exp \left\{-\beta\left(E_{v_{e}}-\mu_{v_{e}}^{\text {eq. }}\right)\right\} 1 / \lambda\left(E_{v_{e}}\right), \mu_{v_{e}}^{\text {eq. }}=\mu_{e}-\left(\mu_{n}-\mu_{p}\right),
$$

with inverse temperature, $\beta=1 / T$. Expression (2.1) is based on the free Fermi gas of individual nucleons. Hence, in the limit of low degeneracy the second term in expression (2.1), which relates to nucleon densities and nucleon degeneracy, must reproduce the density of targets, $n_{n}$ for $v_{e}$ as well as $n_{p}$ for $\bar{v}_{e}$. To recover this low-density limit, the nucleon chemical potentials, $\mu_{N}^{0}$, that enter in Eq. (2.1) must be those of the free Fermi gas. In supernova simulations these quantities are usually provided by the nuclear equation of state (EOS). Nucleons are typically treated in the quasi-particle picture that move in a potential, $U_{N}$, and obey the following relativistic dispersion relations,

$$
E_{N}\left(\mathbf{p}_{N}\right)=\sqrt{\mathbf{p}_{N}^{2}+m_{N}^{* 2}}+U_{N}, \quad(N=\{n, p\}) .
$$

In the case of uniform matter the single-particle potentials are given by the vector self energy in the relativistic mean field framework. They and the effective masses, $m_{N}^{*}$, depend on the independent variables baryon density $\rho$, temperature $T$, and electron fraction $Y_{e}$. The nuclear chemical potentials which enter Eq. (2.1) are corrected accordingly, i.e. $\mu_{N}^{0}=\mu_{N}-U_{N}-m_{N}^{*}$ where $\mu_{N}$ are the full potentials that contain contributions from interactions. Since neutrino transport uses reaction rates with respect to the incoming (anti)neutrino energy, $E_{v_{e}}\left(E_{\bar{v}_{e}}\right)$, one can apply energy conservation and 
use the particle's dispersion relations Eq. (2.3) to relate them with the electron(positron) energy, $E_{e^{-}}\left(E_{e^{+}}\right)$, in the zero-momentum transfer approximation:

$$
E_{v_{e}}=E_{e^{-}}-\left(m_{n}-m_{p}\right)-\triangle U, \quad E_{\bar{v}_{e}}=E_{e^{+}}+\left(m_{n}-m_{p}\right)+\triangle U
$$

with $\triangle U=U_{n}-U_{p}$. This quantity is related to the nuclear EOS, in particular to the nuclear symmetry energy which can be deduced form the parabolic expansion,

$$
\mu_{n}\left(Y_{e}, T, \rho\right)-\mu_{p}\left(Y_{e}, T, \rho\right)=4\left(1-2 Y_{e}\right) S_{B}^{F}(T, \rho),
$$

where $S_{B}^{F}$ is the free symmetry energy associated with the free energy due to finite temperatures. Hence, there is a direct proportionality between nucleon potential differences and nuclear symmetry energy and there is a direct impact of the nuclear EOS on the (anti)neutrino absorption opacity in terms of these medium modification $[24,25]$. Note that for the inelastic treatment of these weak processes, this simply analysis breaks down and a more complicated dependence of the medium modifications is recovered [9].

Fig. 2 shows the opacity of the weak processes (1), (2) and (8) at selected conditions. In addition, we also introduce a new source of $\bar{v}_{e}$-opacity due to the inverse neutron decay obtained from expression (2.1) with the following settings:

$$
\bar{v}_{e}+e^{-}+p \longrightarrow n: \quad\left(1-f_{e}\right) \longrightarrow f_{e} \quad, \quad E_{\bar{v}_{e}}=-E_{e^{-}}+\left(m_{n}-m_{p}\right)+\triangle U
$$

It becomes possible only due to the medium modifications, similarly as they suppress the $\bar{v}_{e^{-}}$ absorption opacity (see the green dash-dotted line in Fig. 2). From the expressions (2.6) it becomes clear that the opacity has a maximum at $E_{\bar{v}_{e}}=0$ with suppression towards high neutrino energies due to the medium modifications (see Fig. 2).

\section{Nucleosynthesis relevant conditions}

We apply the input physics introduced and simulate the PNS deleptonization phase using the general relativistic radiation hydrodynamics code AGILE-BOLTZTRAN [6] with three-flavor Boltzmann neutrino transport. We start from the $11.2 \mathrm{M}_{\odot}$ pre-collapse progenitor [26] and evolve the innermost $10^{5} \mathrm{~km}$ consistently through core collapse, core bounce and early post-bounce mass accretion phases. During the latter which we enhance the neutrino heating and cooling rates in order to trigger the explosion onset. Once the explosion proceeds we switch back to the standard rates. The first few seconds of this evolution are shown in Fig. 1.

The nucleosynthesis relevant conditions, i.e. the entropy per baryon and the electron fraction, in the neutrino-driven wind are determined from the competition of the weak processes (1) and (2) in Table 1. The corresponding neutrino spectra and fluxes are determined at the neutrino spheres, for which we distinguish two definitions [27, 28]: sphere of last inelastic reaction (vertical black solid lines in Fig. 3) and sphere of last elastic scattering (vertical black dash-dotted lines in Fig. 3). In Fig. 3 we also compare the role of different weak processes for all three flavors from where it becomes clear that for $v_{\mu / \tau}$ and $\bar{v}_{e}$ are dominated by scattering on neutrons. This mainly elastic process defines a large scattering atmosphere above the neutrinosphere of last energy exchange, which is defined from mainly inelastic scattering on electrons and positrons for both flavors. In 


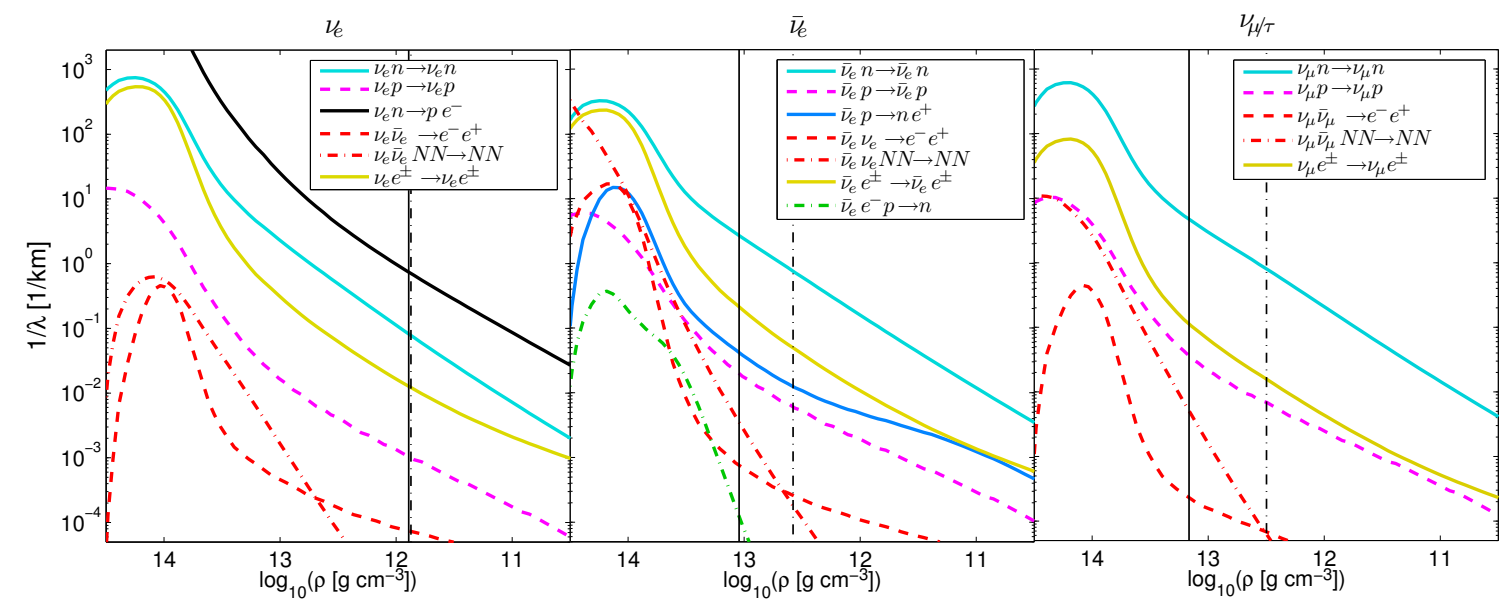

Figure 3: Density dependence of the integrated opacity including final-state Pauli blocking of the neutrinos for selected weak processes during the early PNS deleptonization phase. Vertical black lines mark the position of the neutrino sphere of last elastic (dash-dotted) and inelastic processes (solid).

addition for $\bar{v}_{e}$, absorption on protons plays a subleading role mainly because of the low abundance of protons. The situation is different for $v_{e}$, which are dominated by the charged-current opacity from the absorption on neutrons. Its opacity dominates over any elastic process, and hence a scattering atmosphere has not been developed for $v_{e}$ [29]. In addition to the standard set of weak rates, in Fig. 3 we also show the $\bar{v}_{e}$-opacity from the inverse neutron decay (green dash-dotted line). Here it plays only a subleading role and may become important at later times when the neutrino decoupling region moves to generally higher densities. In particular, it may compensate the suppression of the low-energy $\bar{v}_{e}$-absorptions due to the medium modifications.

The resulting neutrino luminosities and spectra evolution is shown in Fig. 4(a). Even though the fluxes differ only marginally, the average energies reflect the above discussed phenomenology at the level of the neutrino opacity. Weak coupling to matter due to the absence of contributions from charged-current absorption processes result in similarly high neutrino energies for $v_{\mu / \tau}$ and $\bar{v}_{e}$. Their spectra are dominated from elastic scattering on neutrons, a process which does not distinguish between flavors. In contrast, the stronger coupling of $v_{e}$ via charged-current absorption on neutrons results in low average energies for the entire period of about 20 seconds considered here.

The evolution of the neutrino average energies has important consequences for the subsequent nucleosynthesis of heavy elements in the neutrino-driven wind ejecta, in particular the difference between $\left\langle E_{\bar{V}_{E}}\right\rangle$ and $\left\langle E_{V_{E}}\right\rangle$. As material is ejected form the PNS surface due to neutrino heating, these very same processes (reactions (1) and (2) in Table 1) also determine the electron fractions $Y_{e}$. The initially moderately large spectral differences, $\left\langle E_{\bar{v}_{E}}\right\rangle-\left\langle E_{V_{E}}\right\rangle \simeq 4 \mathrm{MeV}$, result in slightly neutron rich conditions between 0.5-3 seconds post bounce (see the lower panel in Fig. 4(b)). $Y_{e}$ rises slowly from $0.47-0.5$, indicating the reducing spectral differences between $\bar{v}_{e}$ and $v_{e}$. During this early PNS evolution most of the mass in the neutrino-driven wind is ejected, with entropies per baryon rising slowly from $S=30-50 \mathrm{k}_{\mathrm{B}}$. It corresponds to the epoch during which the dominant abundance patterns are synthesis, i.e. elements in the region of $\mathrm{Sr}, \mathrm{Y}, \mathrm{Zr}, \mathrm{Nb}$ and $\mathrm{Mo}$ 


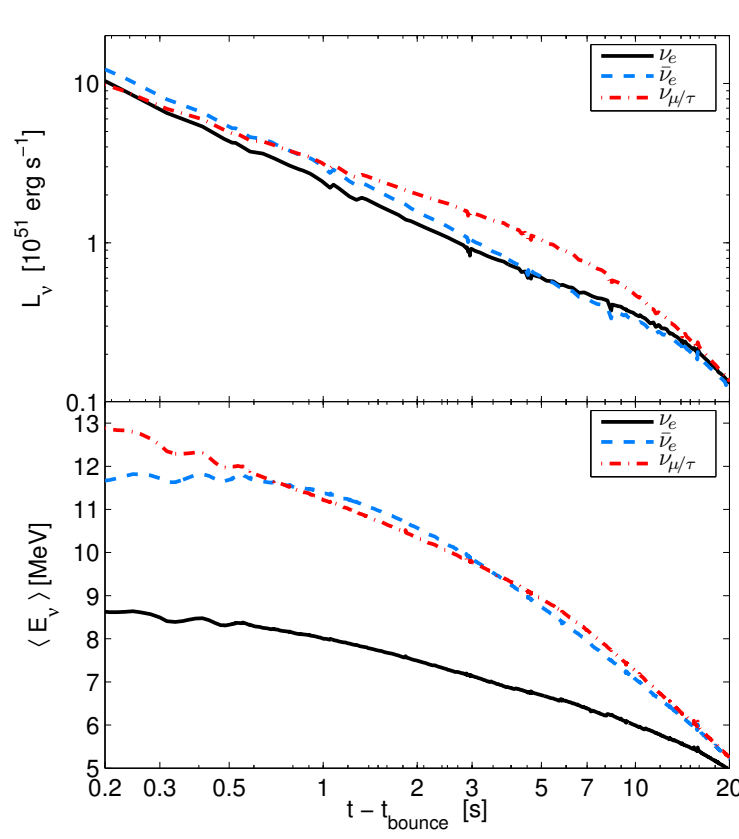

(a) Neutirno luminosities and average energies.

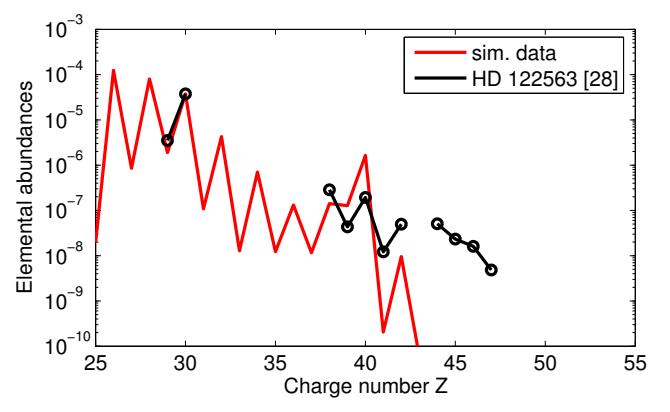

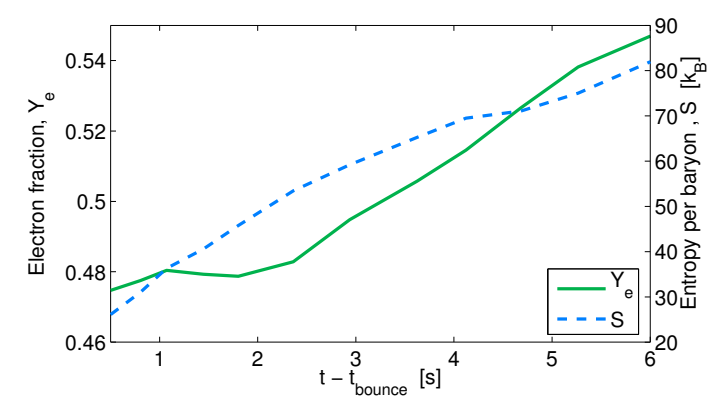

(b) Neutrino-driven wind $Y_{e}$, entropy and abundances.

Figure 4: Evolution of neutrino luminosities and average energies as well as the nucleosynthesis relevant conditions $Y_{e}$ and entropy per baryon, for the $11.2 \mathrm{M}_{\odot}$ simulation. The data shown here correspond to the PNS deleptonization phase after the supernova explosion has been launched. The $Y_{e}$ and entropy values have been obtained using a full nuclear reaction network that includes neutrino interactions on nucleons and nuclei as well as account for the $\alpha$ effect. Furthermore, we also show the total mass-integrated nucleosynthesis abundances normalized to $\mathrm{Zn}(Z=30)$ in comparison to the metal-poor star observations HD 122563 [30].

between $38<Z<45$. It is consistent with observations of metal-poor stars which are deficient in heavy neutron-capture elements beyond $Z>45$ [30]. The small and continuously reducing spectra difference between $\bar{v}_{e}$ and $v_{e}\left(\left\langle E_{\bar{v}_{E}}\right\rangle-\left\langle E_{v_{E}}\right\rangle<2.5 \mathrm{MeV}\right)$ results in proton-rich conditions $Y_{e}>0.5$ at any later time $>3$ seconds (see Figs. 4(a) and 4(b)). It is subject to the $v p$ process with the production of heavy elements with $Z>42(A>92)$ [31-33]. However, their production is very inefficient due to the low (anti)neutrino luminosities and the mass ejected at later times is very small. Hence, a strong imprint from the $v p$ process in the integrated abundance pattern is not observed.

\section{Summary}

We discussed preliminary results from simulations of core-collapse supernovae in spherical symmetry with particular focus on the evolution PNS deleptonization phase. It is determined from the emission of neutrinos of all flavors which drive a low mass-mass outflow known as neutrinodriven wind. We discussed the evolution of the nucleosynthesis relevant conditions in terms of neutrino luminosities and average energies. These can be understood at the level of the opacity, where $v_{\mu / \tau}$ and $\bar{v}_{e}$ are dominated by elastic scattering on neutrons, and have hence very similar spectra. $v_{e}$ are dominated by charged-current absorption on neutrons with a stronger coupling to 
matter and significantly lower average energy. The resulting nucleosynthesis of heavy elements beyond iron produces light neutron-capture elements between $38<Z<42$ in slightly neutron-rich environment during the early evolution up to few seconds during which most mass is ejected.

The here presented preliminary results are based on the nuclear EOS HS.DD2. It has a direct impact on the weak charged-current absorption rates on nucleons for $v_{e}$ and $\bar{v}_{e}$ via the medium modifications related to the nuclear symmetry energy and its density dependence, a nuclear matter property which is currently highly uncertain in matter with large neutron excess. It can be understood that a magnitude of neutron excess scales with the symmetry energy. Thereby of relevance is the region of the neutrino decoupling form matter. Furthermore, the medium modifications allow for the inverse neutron decay as additional channel for the $\bar{v}_{e}$-opacity, which has not been included in the current simulations. This may change the nucleosynthesis relevant conditions in particular at late times when the $\bar{v}_{e}$ charged current absorption rates become suppressed.

\section{Acknowledgement}

TF acknowledges support from the Narodowe Centrum Nauki (NCN) within the "Maestro" program under contract No. DEC-2011/02/A/ST2/00306. GMP and LH are partly supported by the Deutsche Forschungsgemeinschaft through contract SFB 634, the Helmholtz International Center for FAIR within the framework of the LOEWE program launched by the state of Hesse and the Helmholtz Association through the Nuclear Astrophysics Virtual Institute (VH-VI-417). The supernova simulations were performed at the SUPERNOVA computer cluster of the Wroclaw Centre for Networking and Supercomputing (WCSS).

\section{References}

[1] J. M. LeBlanc and J. R. Wilson, Astrophys.J., 161, 1970

[2] A. Burrows, E. Livne, L. Dessart, C. D. Ott, and J. Murphy, Astrophys.J., 640, 878, 2006

[3] I. Sagert, T. Fischer, M. Hempel, G. Pagliara, J. Schaffner-Bielich, et al, Phys.Rev.Lett., 102, 081101, 2009

[4] H. A. Bethe and R. J. Wilson, Astrophys.J., 295, 14, 1985

[5] H.-T. Janka. Annual Review of Nuclear and Particle Science, 62, 407, 2012

[6] M. Liebendörfer, O.E.B. Messer, A. Mezzacappa, S.W. Bruenn, C.Y. Cardall, et al, Astrophys.J.Suppl., $150,263,2004$

[7] T. Fischer, S.C. Whitehouse, A. Mezzacappa, F.-K. Thielemann, and M. Liebendörfer, Astron.Astrophys., 517, A80, 2010

[8] L. Hüdepohl, B. Müller, H.-Th. Janka, A. Marek, and G. G. Raffelt, Phys. Rev. Lett., 104, 251101, 2010

[9] S. Reddy, M, Prakash, and J. M. Lattimer, Phys. Rev., D58, 013009, 1998

[10] A. Juodagalvis, K. Langanke, W. R. Hix, G. Martínez-Pinedo, and J. M. Sampaio, Nuclear Physics A, $848,454,2010$ 
[11] S. W. Bruenn, Astrophys.J.Suppl., 58, 771, 1985

[12] A. Mezzacappa and S. W. Bruenn, Astrophys.J., 405, 637, 1993

[13] A. Mezzacappa and S. W. Bruenn, Astrophys.J., 410, 740, 1993

[14] S. Hannestad and G. G. Raffelt, Astrophys.J., 507, 339, 1998

[15] R. Buras, H.-Th.Janka, M. T. Keil, G. G. Raffelt, and M. Rampp, Astrophys.J., 587, 320, 2003

[16] T. Fischer, S. C. Whitehouse, A. Mezzacappa, F.-K. Thielemann, and M. Liebendörfer, Astronom.Astrophys., 499, 1, 2009

[17] S. E. Woosley, J.R. Wilson, G.J. Mathews, R.D. Hoffman, and B.S. Meyer, Astrophys.J., 433, 229, 1994

[18] Y. Z. Qian and S.E. Woosley, Astrophys.J., 471, 331, 1996

[19] K. Langanke, G. Martínez-Pinedo, B. Müller, H.-Th. Janka, A. Marek, et al., Phys. Rev. Lett., 100, 011101, 2008

[20] T. Fischer, K. Langanke and G. Martínez-Pinedo, Phys. Rev., C88, 065804, 2013

[21] C. J. Horowitz, Phys. Rev., D65, 043001, 2002

[22] M. Hempel and J. Schaffner-Bielich, Nucl.Phys., A837, 210, 2010

[23] S. Typel, Phys. Rev., C71, 064301, 2005

[24] G. Martínez-Pinedo, T. Fischer, A. Lohs, and L. Huther, Phys. Rev. Lett. 109, 251104, 2012

[25] L. F. Roberts, S. Reddy, and G. Shen, Phys. Rev., C86, 065803, 2012

[26] S. E. Woosley, A. Heger, and T. A. Weaver, Rev.Mod.Phys., 74, 1015, 2002

[27] G. G. Raffelt, Astrophys.J., 561, 890, 2001

[28] M. T. Keil, G. G. Raffelt, and H.-T. Janka, Astrophys.J., 590, 971, 2003

[29] T. Fischer, G. Martínez-Pinedo, M. Hempel, and M. Liebendörfer, Phys. Rev., D85, 083003, 2012

[30] S. Honda, W. Aoki, Y. Ishimaru, S. Wanajo, and S. G. Ryan, Astrophys.J., 643, 1180, 2006

[31] C. Fröhlich, Gabriel Martinez-Pinedo, M. Liebendorfer, F.-K. Thielemann, E. Bravo, et al, Phys.Rev.Lett., 96, 142502, 2006

[32] J. Pruet, R. D. Hoffman, S. E. Woosley, H.-T. Janka, and R. Buras, Astrophys.J., 644, 1028, 2006

[33] S. Wanajo, Astrophys.J., 647, 1323, 2006 